\title{
Northwest Territories Residential Southern Placement Program: Dislocation and Colonization through 'Care'
}

\begin{abstract}
Alannis McKee, University of British Columbia
Sean A. Hillier, York University

This research traces colonialism and neoliberalism as foundational architecture to health policy in Canada that seeks to erase Indigeneity and disability and secure the dominance of a White settler able-bodied state. This is accomplished through critical analysis of the Residential Southern Placement Program, a health policy from the Northwest Territories, Canada.

Residential Southern Placements are contractual agreements made between the Northwest Territories Department of Health and Social Services and service agencies from southern provinces to provide care to territorial residents with a disability whose needs - according to the Department of Health and Social Services - cannot be met within the territory. We explore how the ostensibly neutral health policy Residential Southern Placements becomes enacted as a violent intervention of erasure that specifically targets Indigenous adults and children with cognitive disabilities - as evidenced through data collected by a Freedom of Information Request- through long-term and, at times, lifelong dislocation from families, communities, and land. In this analysis we position the Residential Southern Placement Program as an intervention that aims to uphold and safeguard a White settler able-bodied vision of Canadian society. This research highlights an ongoing colonial practice with important implications for disability studies and Indigenous health researchers.
\end{abstract}

Keywords: Indigenous, colonization, trauma, disability, schooling, medical travel, dislocation, Northern, institutionalization 


\section{Introduction}

The formation and implementation of health policy cannot be separated from foundational sociopolitical contexts that govern how a policy is enacted. In Canada, the rise of neoliberalism and entrenchment of colonialism are central tenets to existing social and political architecture. These rationalities naturalize and perpetuate existing power structures and position the dominance of a White settler able-bodied State as natural and inevitable. As a result, ostensibly neutral health policies can serve to further cement the subordinate status of marginalized social groups, including Indigenous Peoples and peoples with disabilities.

In the case of health and social care, policies that appear, or are marketed as, benevolent in nature are often deployed as apparatuses of violence and oppression. Certainly, the relations between Indigenous and non-Indigenous, and able-bodied and disabled, are consistent with such policy interventions. From sterilizations to Residential Schooling to institutionalization and confinement, Indigenous and disabled bodies have similarly been positioned as the 'fortunate' recipients of benevolent State projects of education, reform, care, and rehabilitation. As interventions into the lives of Indigenous Peoples have intensified, so too has the harm and suffering of Indigenous Peoples, which has, strategically, further justified the 'help' these communities continue to require from White settler society. However, despite their benevolent branding, interventions to 'improve' the

\footnotetext{
${ }^{1}$ Data retrieved from my Access to Information Request indicate that $100 \%$ of individuals in the Residential Southern Placement Program have some kind of cognitive disability and approximately 95\% of residents sent south are Indigenous Peoples. Results from the Freedom of Information Request are discussed in detail in the "Results from Freedom of Information Request" section.
}

lives of disabled people and Indigenous Peoples have often translated to the violent erasure of Indigeneity and disability from a White settler, able-bodied vision of Canada.

This research explores the ways in which we can trace overarching eugenic and colonial imperatives aimed at erasing Indigeneity and disability in Canada to the enactment of a specific health policy from the Northwest Territories - the Residential Southern Placements. Residential Southern Placements are contractual agreements made between the Northwest Territories Department of Health and Social Services and service agencies from southern provinces to provide care to territorial residents with a disability whose needsaccording to the Department of Health and Social Services - cannot be met within the territory. This research explores how the ostensibly neutral health policy of Residential Southern Placements becomes enacted as a violent intervention of erasure that specifically targets Indigenous Peoples ${ }^{1}$ with cognitive disabilities ${ }^{2}$ through longterm and, at times, lifelong dislocation from families, communities, and land. In this analysis, we position the Residential Southern Placement Program as an intervention that aims to uphold a White settler able-bodied vision of Canada and as a masked continuation of colonial projects of dislocation.

\section{Northwest Territories Health System Background}

An organizing logic of dislocation is embedded in the delivery of health and

\footnotetext{
${ }^{2}$ In this paper, I adopt the terminology of "cognitive disability" as this is language utilized within the Department of Health and Social Services as it operates the Residential Southern Placement Program. In this case, cognitive disability broadly refers to a range impairment of the mind that fall under the categories of: intellectual disability, mental illness, and brain injuries, as defined by the Department of Health and Social Services (2017a).
} 
social service programming in the Northwest Territories, including the Northwest Territories Long Term Care Program, which governs Residential Southern Placements. The Northwest Territories Long Term Care Program is a division of the Northwest Territories Department of Health and Social Services that operates to meet the needs of territorial residents whose care needs surpass what can be provided in the home environment (NWT Department of Health and Social Services, 2015a). This program delivers care to a fraction of the 45,000 territorial residents, spread across a landmass of 1.3 million square kilometers and divided into 33 communities (Moffit \& Mercer, 2015). Approximately 50\% of these 33 communities are remote and inaccessible by all-season road and most have a population fewer than 1,000 people (Moffit \& Mercer, 2015). Wide geographic dispersal, low population size, and limited infrastructure development within communities are defining features of healthcare delivery in the Northwest Territories. A centralized service model has been the predominant government response to these challenges. Within this framework, longterm care and health services are delivered through regional centres, to which residents from surrounding smaller communities must travel (Young \& Chatwood, 2011). That said, the range of services offered within the territory, even in more largely populated regional centers like the territorial capital, remain highly limited (Young \& Chatwood, 2011). In response to this, the Northwest Territories has established and become heavily reliant on contracted health and social care from southern provinces in Western Canada, particularly Alberta (Young \& Chatwood, 2011). In this system, territorial residents are sent on medical travel from the Northwest Territories to health and social care facilities in provinces to the south where their health needs can be met. Residents with lifelong or long-term care needs that cannot be met in the Northwest Territories are those who become subject to Residential Southern Placements.

Residential Southern Placements are deployed within a context of long-term care service configuration. Since 2009, a singleentry point for all Long-Term Care services exists through a unified Territorial Admissions Committee (TAC) that reviews all applications and makes an offer of a support scheme for each applicant (Fraser, 2011). Limited information about the TAC exists publicly, however, membership on the TAC includes two representatives from Seniors and Continuing Care Services Division, a public representative who serves a two-year term, and one representative from each of the three Health and Social Services Authorities who are nominated by their CEO for a two-year term, and appointed by the Deputy Minister of Health and Social Services. With this change, region-specific health authorities were replaced by a centralized system operating out of Yellowknife. The TAC receives every application for long-term care and holds absolute authority over the provision of services to Northwest Territories residents both within the territory and in care arrangements made with out-of-territory agencies. In the decision-making pathway of the TAC, an application for long-term care is completed and received by a committee that decides whether long term care is necessary or not. If long-term care is deemed necessary, the TAC then decides where the applicant will receive support. If a suitable space - as determined only by the TAC - is available within an individual's region, they are automatically placed there. However, if no suitable space is available within the individual's region, the TAC offers the 'choice' of an alternate facility either within the territory or through a Residential Southern Placement. If the 
individual accepts the 'choice' given to them by the TAC, they gain priority access to that facility; however, if they decline this 'choice' they are put on the bottom of the waitlist for service in their region. Importantly, the territory only has 13 longterm care spaces within the purview of TAC are specifically for residents with cognitive disabilities. Given that these placements are virtually always full, these applicants find themselves uniformly funneled into Residential Southern Placements.

\section{Methods}

This research began with answering the ostensibly straightforward question of how people with cognitive disabilities from the Northwest Territories are sent to another province for years on end to receive care. However, after an initial investigative process, the research purpose evolved from that of quasi-investigative journalism, describing precisely what Residential Southern Placements are and the ways in which they are imposed, to a more critical scholarly question of how this policy is rationalized and enacted onto very specific bodies; namely Indigenous adults and children with cognitive disabilities.

To gather more information about the demographics of individuals subjected to Residential Southern Placements, a Freedom of Information Request (FIR) was submitted to the Access to Information and Health Privacy Officer at the Northwest Territories Department of Health and Social Service. The request was submitted on December 12, 2018 and requested data regarding the age, disability type, community of origin, and race (broken down to Indigenous or nonIndigenous status) for adult placements in the Residential Southern Placement Program. This FIR provided additional data including length of placements, contracted agencies, and contract amounts that were utilized, however, not all of which are addressed in this paper. Public government financial documents were also consulted throughout the analysis to contextualize and supplement data.

Based on the data received from the FIR request, it became clear that the overwhelming overrepresentation of Indigenous Peoples within the program demanded specific attention. This oriented the research to the position of de Leeuw et al. (2010) - originally taken with reference to the Child Welfare System in Canadathat asserts that even though health or social programs, such as the Residential Southern Placement Program, may not explicitly address Indigenous Peoples in their mandate or policies, the fact that the vast majority of individuals in the program are Indigenous means their deployment is "invariably implicitly aimed at Indigenous people" (Leeuw et al., 2010, p. 290). By acknowledging the foundational role of race in the operation of the Residential Southern Placement Program, researchers can explore how this health policy functions as an apparatus of colonialism that advances Indigenous erasure through: the reproduction and perpetuation of dislocation from land imposed onto Indigenous Peoples; disruption of natural connectedness of Indigenous Peoples through the forced separation of families and communities; and disruption of knowledge transmission that impedes the acquisition of traditional languages and cultural expression. The authors of this study come to this work with their own unique worldviews and experiences that has helped to inform this research. McKee is a white settler living with a disability who relocated from southern Ontario to the Northwest Territories as a young child and has lived there with her family ever since. Hillier is a queer mixed Mi'kmaw / settler person who grew up economically disadvantaged in a small isolated community in rural 
Newfoundland whose work focuses on how system level issues impact Indigenous Peoples health.

As the data from the FIR substantially represented Indigenous Peoples, Indigenous community organizations in the Northwest Territories were approached to share and discuss the findings. During the outreach process, ten Indigenous organizations were provided with an infographic of key research findings and demographic data, and representatives from these organizations were invited to set up a time to call or meet about the results. Discussion with organizations included any questions they had regarding the research or the data uncovered, how these findings may impact their organization or the communities they represent, and what action they would like to see in response to the findings. Over the course of May and June 2019, outreach with the ten Indigenous organizations in the Northwest Territories took place. From this, a phone call with four of these organizations and an in-person meeting with one organization was coordinated, whose scope encompassed First Nation, Metis, and Inuit residents from all regions of the territory.

In addition to presenting data gathered with Indigenous organizations, there was a discussion about the ways they would like to see this information mobilized. From these conversations, additional data was requested regarding the number of children in Residential Southern Placements and a breakdown of all individuals in placements by region and First Nation, Inuit, and Metis status. These data sets were requested as a secondary FIR on May 27, 2019 to the Access to Information and Health Privacy Officer at the Northwest
Territories Department of Health and Social Service. Responses to this request were received on June 28, 2019 and July 15, 2019. It was requested by the community organizations that any new data be provided to them so that they may use it as evidence in their own requests for funding or to redistribute control and autonomy over the delivery of health and social services from the Government of the Northwest Territories to Indigenous organizations. This information sharing was completed after each of the discussions.

\section{Results}

Data received through FIR indicate that 162 Northwest Territories residents are in Residential Southern Placements, as of June 2019. Of these, 56 individuals are children ( $<18$ years of age) and 106 are adults ( $>18$ years of age). $89 \%$ of children in Residential Southern Placements have registered Indigenous status and 90\% of adults in Residential Southern Placements have registered Indigenous status, as detailed in Table 1. It should be noted that the FIR request received indicated that, "while the table demonstrates that six children/youth are non-Indigenous this should be interpreted cautiously. The siblings and/or parents of these children/youth are Indigenous. Therefore, based on the ethnicity of siblings and/or parents, some children may not have applied for their Nunavut Inuit Enrolment Card (NTI), Metis Status Card, or First Nation Status Card." The FIR received for adult placements gave no specification on the Indigenous relations of those codified as 'non-Indigenous'. 


\section{Table 1}

Breakdown of Individuals in the Southern Residential Placement Program by Ethnicity as of June 2019

\begin{tabular}{|c|c|c|}
\hline Ethnicity & $\begin{array}{c}\text { Number of Children }(<18 \\
\text { yrs.) in Placement }\end{array}$ & $\begin{array}{c}\text { Number of Adults (> 18yrs.) } \\
\text { in Placement }\end{array}$ \\
\hline First Nation & 35 & 56 \\
\hline Inuit & 10 & 38 \\
\hline Metis & 5 & 1 \\
\hline Non-Indigenous & 6 & 11 \\
\hline Total & 56 & 106 \\
\hline
\end{tabular}

As of October 2019, data on the length of Residential Southern Placements had only been received for adult placements. Data received indicate that placements were predominantly long-term and, in some cases, lifelong. Only $15 \%$ of adult residents in placements had been outside of the territory for less than one year; $32 \%$ of adults had been in their placement for 4-10 years; and $23 \%$ had been in their placement for over 10 years, as outlined in Table 2 .

\section{Table 2}

Length of Residential Southern Placement for Adults as of December 2018

\begin{tabular}{|l|c|c|}
\hline Length of Placement Range & $\begin{array}{c}\text { Approximate Number of } \\
\text { Adults in Range }\end{array}$ & $\begin{array}{c}\text { Percentage of Adults in } \\
\text { Range }\end{array}$ \\
\hline Less than 1 year & 16 & $15.2 \%$ \\
\hline 1-2 years & 10 & $9.5 \%$ \\
\hline 2-4 years & 21 & $20.0 \%$ \\
\hline 4-10 years & 34 & $32.4 \%$ \\
\hline 10-15 years & 8 & $7.6 \%$ \\
\hline 15-20 years & 14 & $13.5 \%$ \\
\hline Greater than 20 years & 2 & $1.9 \%$ \\
\hline
\end{tabular}

Review of the 2017 Request of Proposals for the Residential Southern Placement Program from the Northwest Territories Department of Health and Social Services indicate that $100 \%$ of individuals in the program had some form of cognitive disability, including intellectual disabilities, psychiatric disabilities, and brain injuries, as outlined in Table 3. 
Table 3

Distribution of Residential Southern Placements by Disability Type as of 2017

\begin{tabular}{|l|c|}
\hline \multicolumn{1}{|c|}{ Disability Category } & Percentage of Placements \\
\hline Intellectual disability & $66 \%$ \\
\hline Psychiatric disability & $31 \%$ \\
\hline Brain injury & $3 \%$ \\
\hline
\end{tabular}

According to Northwest Territories Department of Finance budgets, the budget for the Residential Southern Placement Program has increased steadily, such that between 2011 and 2017, government investment in these placements increased from 16.1 million dollars to 28.5 million dollars (a 77\% increase), and now accounts for over $50 \%$ of the Northwest Territories Department of Health and Social Services' total spending on residential care (NWT Department of Finance, 2017a, 2017b; NWT Department of Health and Social Services, 2013, 2014, 2015, 2016, 2017b).

\section{Discussion}

\section{Settler Colonialism and Indigenous Elimination}

Given that a majority of the Northwest Territories population $(50.7 \%)$ is Indigenous, analysis of any of its health policies would be incomplete without consideration of historic and ongoing colonialism that shape the lived experiences of Indigenous Peoples and govern the social landscape where health policy operates (NWT Bureau of Statistics, 2016).

The relationship between the Canadian State and Indigenous Peoples has been, and continues to be, shaped by policy with an unchanging goal to "ensure the eventual disappearance of Indians" (Palmater, 2014, p. 27). This goal of eliminating Indigenous Peoples from what is now Canada has been enacted through the violent intervention of the State into nearly every aspect of Indigenous People's bodies, lives, communities, and lands. While this attempted erasure has taken on innumerable manifestations since initial contact between Indigenous Peoples and White settlersfrom outright state-sanctioned killing through incentivized scalping of Indigenous Peoples, to involuntary sterilization, to violent and aggressive assimilative projects - the rationality driving this elimination, in all adopted methodologies, continues to fall back to the domination and ownership of land (Milloy, 2008; Palmater, 2011; Palmater, 2014). Settler colonialism is a violent enterprise of erasure that asserts the natural domination and ownership of settlers over land and resources controlled by a preceding, and still present, sovereign peoples; in essence, as described by Wolfe (2006), "settler colonialism destroys to replace" (p.388). In this formulation, Indigenous Peoples, with preceding ownership and connection to land, embody a permanent contestation towards the existing domination of the White settler State and, furthermore, an obstacle to the unobjectionable assertion of settler dominion over stolen land. Wolfe (2006) theorizes the settler response to this tension as taking on a particular form of elimination that negatively seeks to destroy Indigenous society through the dissolution of social structures, culture, and political power, and positively seeks to erect a new colonial society (i.e., the White settler state) on its stolen territory. 
In Canada, the attempted elimination of Indigenous societies for the purposes of securing permanent access to Indigenous lands and resources by the settler population has arisen from violently assimilative policies and projects that seek to erase Indigeneity through "absorption into the general population" for purposes of "overcoming lingering native custom and tradition" (Duncan Campbell Scott, as cited in Palmater, 2014, p. 31). From this vantage point, the Canadian State positioned-and continues to position through the presentday enactment of historic policyIndigenous Peoples and cultures as a threat to the actualization of the settler State. This perceived threat is neutralized by the State through heinous colonial projects that are often not advertised as such publicly, at least not explicitly. Since the enactment of the Indian Act in 1869, the violent intervention of the State into the bodies, lives, communities, and lands of Indigenous Peoples has been rationalized as social projects of benevolent transformation that aim to 'help' and 'improve' Indigenous Peoples.

\section{Projects of Elimination in the Northwest Territories}

In northern Canada, and the Northwest Territories specifically, social projects of 'improvement' often rely on a model of dislocation that necessitates the movement of Indigenous Peoples from their home communities to regional centres where care is delivered. Moreover, for people with long-term care needs, particularly those with cognitive disabilities, this dislocation of people from the Northwest Territories is intense, with placements for care outside the territory in southern provinces.

The legacy of trauma faced by people with cognitive disabilities sent south for care can be traced back to historical patterns of dislocation during the era of institutionalization. Once institutionalized in the south, northern people with cognitive disabilities faced increased barriers to remain connected with their home communities and families due to the sheer extent of physical separation, which would have required a high level of independent wealth to overcome. While families of southern people with cognitive disabilities relocated to institutions within their home province may be able, with difficulty, to save enough money to make a trip by road or rail, it is doubtful that families of northern people sent south can ever save enough money to make such a journey, considering the significant cost of transportation by plane and already high cost of living in the north (Menzies \& Palys, 2006). In addition to the increased isolation and emotional devastation experienced by northern people with cognitive disabilities in general, it is also crucial to recognize that the interlocking social identity of Indigeneity would have greatly influenced the experience of southern institutionalization for Indigenous Peoples with cognitive disabilities. Upon institutionalization, Indigenous Peoples with cognitive disabilities became subjected to an additional level of segregation, surveillance, and attempted erasure through the entrenched colonialism of Canada's health system. Segregation of Indigenous persons in medical institutions was commonplace in Canada, especially western and northern Canada, into the 1960s. In Yellowknife's own hospital, where some people with cognitive disabilities were held for a time before being relocated to institutions in the provincial south, Indigenous patients were relegated to 'Indian Wards' until at least 1965 (Lux, 2016). Considering the pervasiveness of the residential school system in the Northwest Territories, with the last school closing in 1994, it is likely that at least some Indigenous Peoples living in the 
north with cognitive disabilities were subjected to the abovementioned process of dislocation and re-traumatization, having been forced out of their communities to attend residential schools and then, again, forced into segregate health institutions in the south. Beyond the already morbid prospects of displacement, illness, poor living conditions, and abuse faced by all people with cognitive disabilities, being institutionalized as an Indigenous person would have also been a profoundly alienating experience (Menzies \& Palys, 2006). Once confined to an institution, it was not uncommon for a patient to discover that they were the only Indigenous person in their ward, or in an entire facility (Menzies $\&$ Palys, 2006). Not only would this have created a significant barrier to cultural expression and sense of belonging, but day to day social life of Indigenous residents may have also been impacted by the fact that English was very often not their first spoken language - if it was spoken at all - and, as such, basic communication was impeded (Menzies \& Palys, 2006). As well, outside the walls of institutions, colonialism and medicalization interlocked in other eugenic projects, such as sexual sterilization, increasing the vulnerability of Indigenous Peoples with cognitive disabilities from the Northwest Territories housed in institutions of the south. For example, from 1969-1972 in Alberta, First Nations people accounted for $25 \%$ of patients sterilized through the Sexual Sterilization Act, despite only representing $3 \%$ of the province's population (Stote, 2012). Together, these factors contributed to an especially violent and painful experience of southern institutionalization for Indigenous Peoples from the north with cognitive disabilities, who not only faced significant geographical separation, but also cultural repression and greater levels of subjugation, isolation, and attempted erasure (Roman et al., 2009).
Importantly, this should not be understood as an issue of the past. Physical distance between families separated through southern placements continues to be an insurmountable barrier for many. This is especially true for Indigenous Peoples, given that the Northwest Territories has the second highest rate of poverty in Canada at $16.3 \%$, and particularly for Indigenous Peoples and those in smaller and remote communities, where the rate of family poverty ranges from $22 \%$ to $50 \%$ (NWT Bureau of Statistics, $2016 b)$. It is difficult to conceptualize the degree of isolation imposed onto northern people with cognitive disabilities, who, during this era of institutionalization, have been treated as limitlessly portable and depersonalized sites of medical management, relegated to unfamiliar lands and climates, a world away from their families and homes with no end in sight.

\section{Residential Southern Placements and Indigenous Elimination through Dislocation}

Given the legacy of dislocation imposed onto Indigenous Peoples in general, it is no surprise that the data from the FIR reveal a disproportionate number of Indigenous Peoples with cognitive disabilities being subjected to Residential Southern Placements. Despite accounting for $50.7 \%$ of the population of the territory, Indigenous residents account for at least $90 \%$ of persons placed outside of the territory for care. It is also clear that this figure is under-representative of the true proportion of individuals in Residential Southern Placements, given that at least 4\% of placements outside the territory are codified 'non-Indigenous' by the Department of Health and Social Services despite a known Indigenous parent or sibling. Of the Indigenous persons in the Residential Southern Placement Program, $100 \%$ of them had some form of cognitive 
disability. Despite a policy that does not specifically address Indigenous Peoples or cognitive disability, it is critical to understand how almost all individuals in these placements fall into both categories. We posit that dislocation is a mechanism of erasure with shared historic and contemporary deployment onto Indigenous Peoples and persons living with cognitive disabilities in Canada.

Projects of erasure that impact people with cognitive disabilities and Indigenous Peoples in settler-colonial Canada have been similarly predicated on an overarching rationality that some persons are better served through the external management of worthy citizens with power (read as White and able-bodied). Institutionalization of people with cognitive disabilities in Canada arose from eugenic thinking that aimed to protect the nondisabled population from the threat of feeblemindedness and was legitimized through a subtext of care and medicalization of disability (Walmsley \& Johnson, 2017). Through eugenic rationality, people with cognitive disabilities were viewed through a moralistic lens and positioned as sites of risk that needed to be addressed through segregation, surveillance, confinement, and management by nondisabled people and, in particular, the State. To legitimize these interventions by the State, people with cognitive disabilities had to be positioned as inherently inferior and non-autonomous objects of management; sites for improvement and transformation towards hegemonic understandings of rationality and intellectual capacity (Walmsley \& Johnson, 2017). In other words, people with cognitive disabilities ought to be 'cared' for by people without disabilities, who are best suited to define and address their needs. In the Residential Southern Placement Program, this organizing logic remains embedded in a pathway of care distribution that places full autonomy with an external governing body (i.e., the TAC), to make decisions about the lives and bodies of persons with cognitive disabilities. Likewise, since contact, Indigenous Peoples and societies have been similarly characterized as inherently deviant and inferior to that of settler society. Through this logic, White settlers strategically positioned themselves as the rightful and natural trustees of Indigenous Peoples and communities and justified an array of violent and intrusive projects into the lives of Indigenous Peoples (de Leeuw et al., 2010, p. 290). Not unlike the interventions aimed at persons with cognitive disabilities, colonial projects including Residential Schooling and the Sixties Scoop that aimed to erase Indigeneity, have similarly organized around dislocation from family and community.

Dislocation is central to the functioning of the Residential Southern Placement Program. In this program, Indigenous Peoples with cognitive disabilities find themselves subjected to displacement from their families, communities, and land for extended periods of time. As indicated from FIR, these placements are by no means short-term and, for most adults, last from years to a lifetime. As such, it is helpful to understand the Residential Southern Placement Program as a current iteration of a colonial legacy of dislocation for the purposes of elimination, that operates under a guise of 'caring for' Indigenous Peoples through medicalization. In what has been described by some scholars as "medical colonialism", Western medical practices and conceptions of health are deployed and imposed onto Indigenous bodies in ways that undermine the integrity of Indigenous communities by disrupting traditional Indigenous social structures and ways of knowing through enforced assimilation and acquiescence to Western systems (Brown et al., 2012, p. 45). This is 
especially pronounced in the enforcement of Western medical interventions and by making basic medical care contingent on dislocation of Indigenous Peoples from their home communities. The fact that many Indigenous Peoples, particularly Indigenous Peoples living outside urban centres and especially those in northern communities, face a dilemma in health care access that forces them to choose between accessing care or staying in their home communities, is well supported in existing scholarship (see, for example: Allan \& Smylie, 2015; Anderson et al., 2008; Czyzewski, 2006; Kolewaski et al., 2010; Martens, 2000; Moffit \& Vollman, 2006; Reading \& Wien, 2013; Salvalaggio \& Miore, 2003). The disconnection that comes from Indigenous Peoples' need to relocate for access to health care engenders intense loneliness, isolation, alienation, and negative mental and physical health outcomes (Allan \& Smylie, 2015; Auger, 2016; Kolewaski et al., 2010; Salvalaggio \& Miore, 2003; Moffit \& Vollman, 2006). In addition to the devastating impact this has on an individual level, separation from one's land, family, and community also have important implications for Indigenous societies, where fractured families and communities disrupt the intergenerational and lateral transmission of cultural knowledge, language, and identity formation (Reading \& Wied, 2009). Of particular relevance to this paper is specific research identifying that Indigenous Peoples with cognitive disabilities dislocated for the purpose of accessing care are more highly represented in large institutional-like settings, less likely to return to their home communities, and have systemic challenges accessing and engaging with Indigenous language, culture, and community while living in residential settings (Auger, 2016; Rossow-Kimball et al., 2017). This is perhaps best captured through the words of Indigenous Peoples themselves, such as those of a 'Namgis participant in a Brown et al.'s (2012) study of dispossession and health equity, who stated that living away from home territories has the impact of "undermining sacred connections that nourish the relationship between one and another" (p. 55). Where land is the lifeblood of culture and connectedness for Indigenous societies, dislocation serves as a direct attack on the existence of Indigenous societies, and, further, the resistance they pose to the actualization of the White settler State. By orchestrating the dislocation of Indigenous Peoples from their own land, the Canadian State engages in a violent assertion of dominance that implies White settlers have the inherent authority over this land, to choose who can go where, and erase Indigenous sovereignty and history altogether.

What also cannot be ignored is a trajectory towards further investment and dependence on placements of dislocation as a catch-all response for Indigenous adults and children living with cognitive disabilities. As evidenced in review of budgets from the Department of Finance and Department of Health and Social Services, the Government of the Northwest Territories continues to increase its spending on out-ofterritory services such that the budget for out of territory placements has increased $77 \%$ in just 6 years between 2011 and 2016. A similar investment within the territory for non-institutional residential supports for persons with disabilities has been notably absent. Unrestricted and indefinite investment in southern agencies is an approach that has, and continues to, predictably lead to a continued need to move people out of the territory. Existing spending patterns indicate that this destructive system of care is only set to expand moving forward. 


\section{Conclusion}

The Residential Southern Placement Program facilitates contractual care arrangements between the Government of the Northwest Territories and southern care agencies for individuals who, according to the TAC, cannot have their needs met within the territory. Placements are predominantly long-term and impose intense dislocation, literally thousands of kilometers, from one's and home community to agencies in the provincial south.

Although never naming Indigenous Peoples or persons with cognitive disabilities, available data establishes that the Northwest Territories Residential Southern Placement Program is deployed for Indigenous Peoples with cognitive disabilities at least $90 \%$ of the times it is utilized. To understand how Indigenous Peoples with cognitive disabilities became the primary target of this program, this research contextualizes Residential Southern Placements into a history of colonialism that seeks to eliminate cognitive disability and Indigeneity from a White settler, ablebodied vision of what is now Canada. This research highlights an ongoing colonial practice with important implications for disability studies and Indigenous health researchers. This research serves as a critical point of intersectional scholarship and provides a framework for disability communities and Indigenous Peoples to work in collaboration towards the redistribution of power, resources, and justice for all people that call the Northwest Territories home.

\section{References}

Allan, B. \& Smylie, J. (2015). First Peoples, second class treatment: The role of racism in the health and well-being of Indigenous peoples in Canada. Toronto, ON: the Wellesley Institute.

Anderson, K., Yeates, K., Cunningham, J., Devitt, J., \& Cass, A. (2009). They really want to go back home, they hate it here: The importance of place in Canadian health professionals' views on the barriers facing Aboriginal patients accessing kidney transplants. Health \& Place, 15, 390-393.

https://doi:10.1016/j.healthplace.2008.0 3.002

Auger, M. D. (2016). Cultural continuity as a determinant of Indigenous people's health: A metasynthesis of qualitative research in Canada and the United States. The International Indigenous Policy Journal, 7(4). https://doi:10.18584/iipj.2016.7.4.3

Brown, H. J., McPherson, G., Peterson, R., Newman, V., \& Cranmer, B. (2012). Our land, our language: Connecting dispossession and health equity in an Indigenous context. Canadian Journal of Nursing Research, 44(2), 44-63.

Czyzewski, K. (2011). Colonialism as a broader social determinant of health. The International Indigenous Policy

Journal, 2(1), 1-14. https://doi:10.18584/iipj.2011.2.1.5

de Leeuw, S., Greenwood, M., \& Cameron, E. (2010). Deviant constructions: How governments preserve colonial narratives of addictions and poor mental health to intervene into the lives of Indigenous children and families in Canada. International Journal of Mental Health and Addictions, 8, 282-295. https://doi:10.1007/s11469-009-9225-1

Fraser, S. (2011). Northwest Territories health programs and services-2011 Department of Health and Social Services. Report Prepared for Office of the Auditor General of Canada. Ottawa, Canada. https://www.fin.gov.nt.ca/sites/hss/fin/fi les/201718_budget_address_final_pdf.pf 
Kolewaski, C. D., Paterson, M. L., Yeates, K. E. (2010). Relocation from the Mushkegowuk Territory for hemodialysis: The Cree illness experience and perceived quality of life. Pimatisiwin: A Journal of Aboriginal and Indigenous Community Health, 8(3), 103-150.

Lux, M. K. (2016). Making Indian hospitals. In Separate beds: A history of Indian hospitals in Canada, 1920s-1980s (pp. 19-47). Toronto, ON: University of Toronto Press. https://doi.org/10.3138/9781442663114 $-004$

Martens, C. (2000). Intellectual disability and Aboriginal people: An overview of current practice and process in institutionalization [Graduate thesis, University of Manitoba].

http://www.collectionscanada.gc.ca/obj/ s4/f2/dsk1/tape3/PQDD_0022/MQ5176 $0 . p d f$

Menzies, R., \& Palys, T. (2006). Turbulent spirits: Aboriginal patients in the British Columbia psychiatric system, 18791950. In J. E. Morgan \& D. Wright (Eds.), Mental health and Canadian society: Historical perspectives (pp. 149-175). Montreal, Quebec: McGillUniversity Press.

Milloy, J. (2008). Indian Act colonialism: A century of dishonour, 1869-1969.

National Centre for First Nations

Governance Website:

http://fngovernance.org/ncfng_research/ milloy.pdf

Moffit, P. \& Mercer, A. (2015). Northwest Territories. In State of Rural Canada Report. http://sorc.crrf.ca/nwt/

Moffit, M. P., \& Vollman, A. R. (2006). At what cost to health? Tlicho women's medical travel for childbirth.

Contemporary Nursing, 22(2), 228-239. https://doi:10.5172/conu.2006.22.2.228
NWT Bureau of Statics. (2016a). Population projections.

https://www.statsnwt.ca/population/com munity-projections/

NWT Bureau of Statistics. (2016b). Poverty indicators.

https://www.statsnwt.ca/Poverty\%20Ind icators/index.html

NWT Department of Finance. (2017a). Budget main estimates: 2017-2018. https://www.fin.gov.nt.ca/sites/fin/files/ 2017-2018_main_estimates.pdf

NWT Department of Finance. (2017b). Annual business plan 2018-2019 Health and Social Services.

https://www.fin.gov.nt.ca/sites/fin/files/ 2018-

19_annual_business_plans_for_website. pdf

NWT Department of Health and Social Services. (2013). Measuring success and focusing on results: NWT Health and Social Services System 2011-2012 Annual Report.

https://www.hss.gov.nt.ca/sites/hss/files /2011-2012-annual-report-measuringsuccess-and-focusing-results.pdf

NWT Department of Health and Social Services. (2014). Measuring success and focusing on results: NWT Health and Social Services System 2012-2013 Annual Report. https://www.assembly.gov.nt.ca/sites/de fault/files/td_42-175.pdf

NWT Health and Social Services. (2015a). Northwest Territories long-term care program review: Final report. https://www.hss.gov.nt.ca/sites/hss/files /nwt-long-term-care-programreview.pdf

NWT Department of Health and Social Services. (2015b). Measuring success and focusing on results: NWT Health and Social Services System 2013-2014 Annual Report. https://www.hss.gov.nt.ca/sites/hss/files 
/2013-2014-annual-report-measuringsuccess-and-focusing-results.pdf

NWT Department of Health and Social Services. (2016). Measuring success and focusing on results: NWT Health and Social Service System 2014-2015 Annual Report.

https://www.hss.gov.nt.ca/sites/hss/files /measuring-success-focusing-resultshss-annual-report-2014-15.pdf

NWT Health and Social Services. (2017a). Request for proposals: Non-exclusive Out of Territory (OOT) placement program-youth, adult or family services.

NWT Department of Health and Social Services. (2017b). Caring for our people: Strategic plan for the NWT health and social services system 2017 to 2020.

https://www.assembly.gov.nt.ca/sites/de fault/files/td_328-182.pdf

Palmater, P. (2011). Stretched beyond human limit: Death by poverty in First Nations. Canadian Review of Social Policy, 65/66, 112-127.

Palmater, P. (2014). Genocide, Indian policy, and legislated elimination of Indians in Canada. Aboriginal Policy Studies, 3(3), 27-54. https://doi:10.5663/aps.v3i3.22225

Reading, C., \& Wien, F. (2009). Health inequalities and social determinants of Aboriginal peoples' health [Report]. https://www.ccnsanccah.ca/docs/determinants/RPTHealthInequalities-Reading-WienEN.pdf

Roman, L.G., Brown, S., Noble, S., Wainer, R., \& Young, A. E. (2009). No time for nostalgia!: Asylum-making, medicalized colonialism in British Columbia (1859-97) and artistic praxis for social transformation. International Journal of Qualitative Studies in Education, 22(1), 17-63. https://doi.org/10.1080/0951839080258 1919

Salvalaggio, G., Len, K., \& Miore, B. (2003) Perspective on health: Experiences of First Nations dialysis patients relocated from remote communities for treatment. Canadian Journal of Rural Medicine, 8(1), 19-24.

Stote, K. (2012). The coercive sterilization of Aboriginal women in Canada. American Indian Culture and Research Journal, 36(3), 117-150. https://doi.org/10.17953/aicr.36.3.72807 28r6479j650

Walmsley, J., \& Johnson, K. (2017). Institutionalizing rural life. In $\mathrm{K}$. Soldatic \& K. Johnson (Eds.), Disability and rurality: Identity, gender and belonging (pp. 53-68). New York, NY: Routledge. https://doi.org/10.4324/9781315577340 $-5$

Wolfe, P. (2006). Settler colonialism and the elimination of the native. Journal of Genocide Research, 8(4), 387-409. https://doi:10.1080/1462352060105624 0

Young, T. K., \& Chatwood, S. (2011). Health care in the North: What Canada can learn from its circumpolar neighbours. Canadian Medical Association Journal, 183(2), 209-214. https://doi:10.1503/cmaj.100948 